\title{
Comparison of the Effect of Edible Citrullus colocynthis Fruit Powder with Metformin on the Level of Blood Glucose in Streptozotocin-Induced Diabetic Male Rats
}

\author{
Mojaz Dalfardi N. ${ }^{1}$ PhD, Ghodrati Azadi H.* PhD, Fathi Hafshjani B. ${ }^{1}$ PhD
}

*Basic Sciences Department, Veterinary Medicine Faculty, Ferdowsi University of Mashhad, Mashhad, Iran

${ }^{1}$ Basic Sciences Department, Veterinary Medicine Faculty, Ferdowsi University of Mashhad, Mashhad, Iran

\begin{abstract}
Aims: Diabetes is one of the metabolic diseases. According to side effects of medication and high costs of treatments, medical plants are of attention. This study was done to compare the anti-diabetic effect of Citrullus colocynthis fruit powder with metformin on blood glucose level.

Materials \& Methods: 32 mature male rats with an average weight of 250$300 \mathrm{gr}$ were divided into four groups; a negative control (normal diet), a positive control (distilled water), a sham (14 mg/kg metformin) and a treatment $(50 \mathrm{mg} / \mathrm{kg}$ Citrullus colocynthis). Type 2 diabetes induced by intraperitoneal injection of $60 \mathrm{mg} / \mathrm{kg}$ of Streptozotocin (two times, with $48 \mathrm{~h}$ interval) in all except the negative control group. These solutions regularly administrated by using oral gavage for 6 weeks. After 12 hours of fasting, before the first and after the last doses, the blood glucose was measured via the tail vein. Statistical analysis was performed in SPSS 18 software by one way ANOVA with repeated measurement tests.

Findings: The decreasing level of glucose between metformin and Citrullus colocynthis groups was significant in day $28(\mathrm{p}<0.05)$, But it was not significant in day 42.

Conclusion: Metformin can be replaced by Citrullus colocynthis in reducing blood glucose.

Keywords

Diabetes Mellitus [http://www.ncbi.nlm.nih.gov/mesh/68003920];

Citrullus colocynthis [http://www.ncbi.nlm.nih.gov/mesh/68065986];

Metformin [http://www.ncbi.nlm.nih.gov/mesh/68008687];

Streptozocin [http://www.ncbi.nlm.nih.gov/mesh/68013311];

Blood Glucose [http://www.ncbi.nlm.nih.gov/mesh/68001786]
\end{abstract}


اختلال در عملكرد اركانهاى متعددى بهويزه جشمها، كليهها، اعصاب، قلب و رَّهاى خونى همراه است.

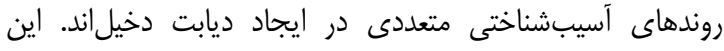

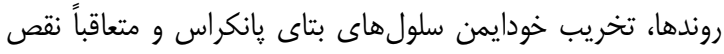

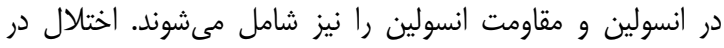

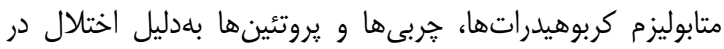

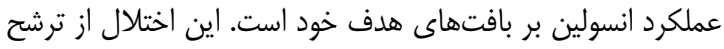

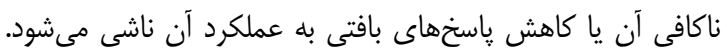

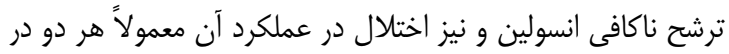

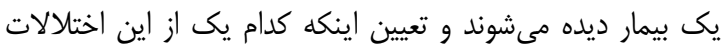

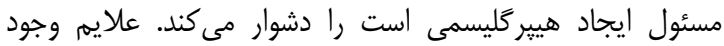

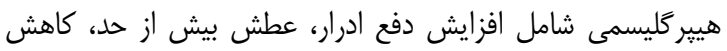
وزن، در برخى موارد اشتهاى زياد و تاربينى است [1]

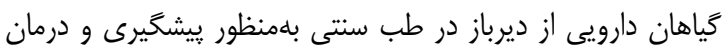

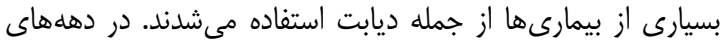

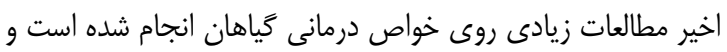

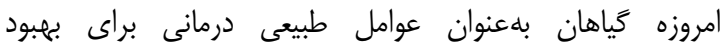
بيمارىهاى مختلف مطرح شدهاند [؟].

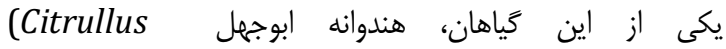

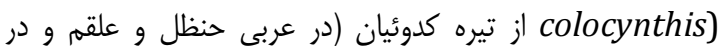

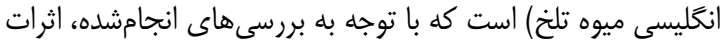

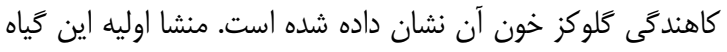

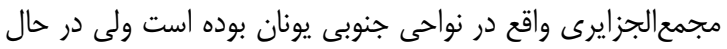

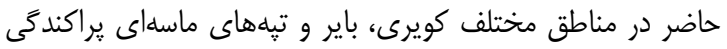
دارد و در مناطق مختلف ايران نيز مانند لرستان، اهواز، كازرون،

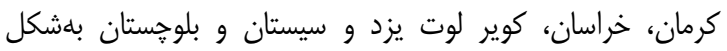

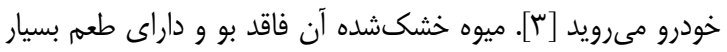

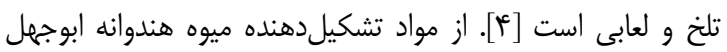

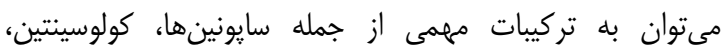

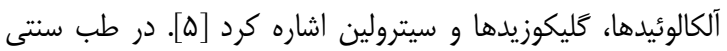

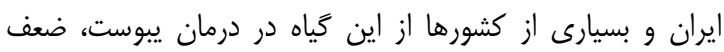

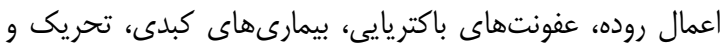

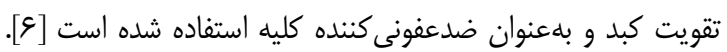

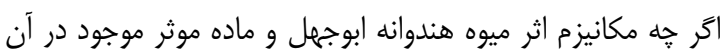

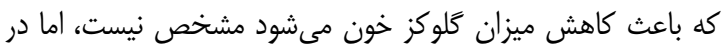

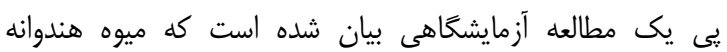

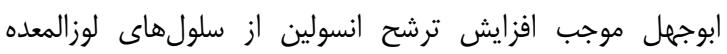

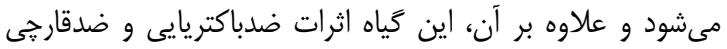

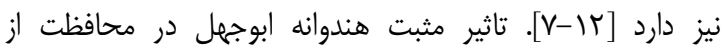
سلولهاى لوزالمعده و ييشخيرى از اختلالات متابوليزمى ناشى از

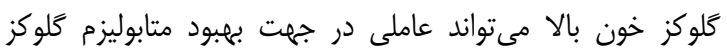
خون و در نتيجه كاهش آن باشد [سان]

\section{مقايسه تاثير خوراكى يودر ميوه هندوانه ابوجهل

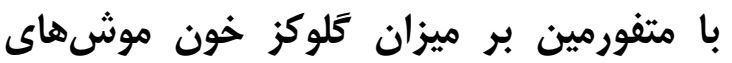 صحر ايیى نر ديابتىشده با استر يتوزوسين}

نسيم مجاز دلفاردى PhD

كروه علوم بايه، دانشكده داميز شكى، دانشكاه فردوسى مشهرد، مشهد، ايران

حميده قدر تى آزادى " PhD

كروه علوم پايه، دانشكده داميزشكى، دانشعاه فردوسى مشهرد، مشهل، ايران

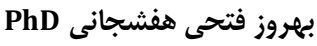

كروه علوم يايه، دانشكده داميزشكى، دانشكاه فردوسى مشهد، مشهد، ايران

جكيده

اهداف: ديابت، از شايعترين بيمارىهاى متابوليك است. بهعلت عوارض

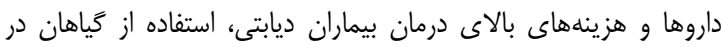

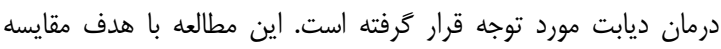

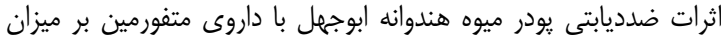

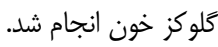

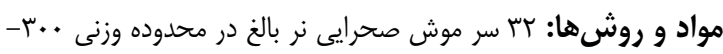

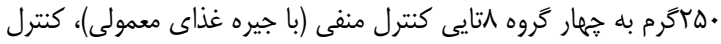

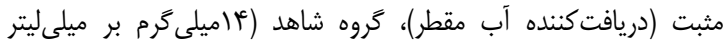

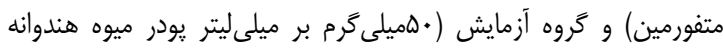

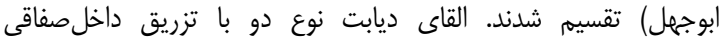

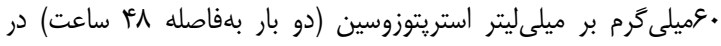

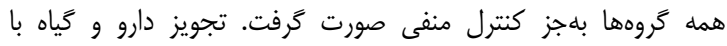

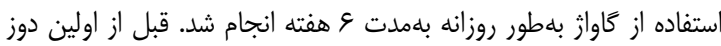

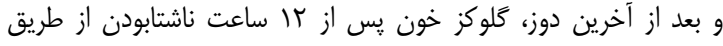

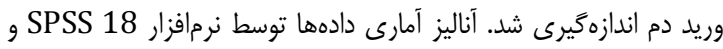

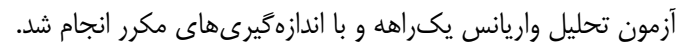

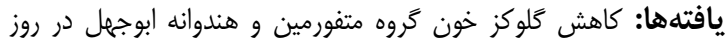

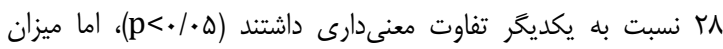

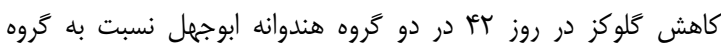
متفورمين معنى دار نبود.

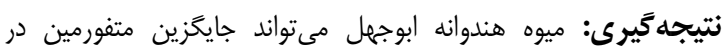

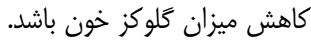
كليدوازهها: ديابت، هندوانه ابوجهل، متفورمين، استريتوزوسين، كلوكز خون

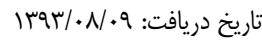

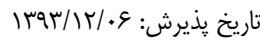
ghodrati@um.ac.ir :

مقدمه - - مق

ديابت اختلالى متابوليك است كه بلوسيله هيير كليسمى (كه بهدليل

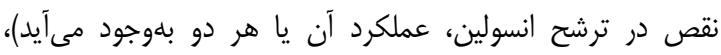

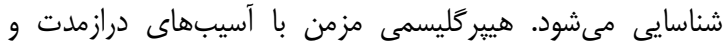




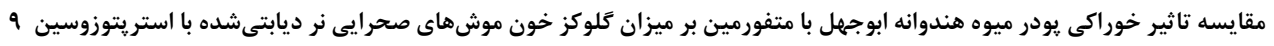

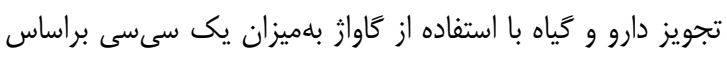

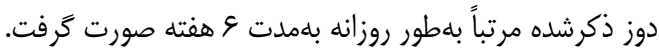

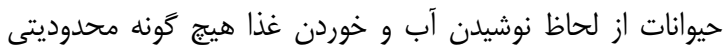

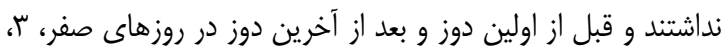

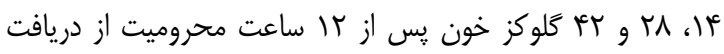

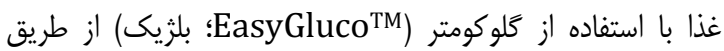

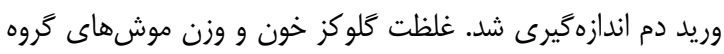

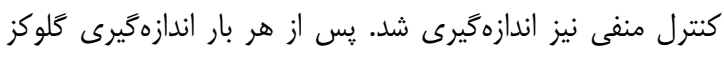

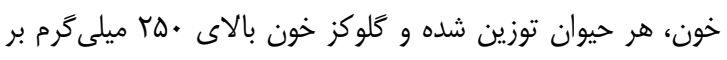

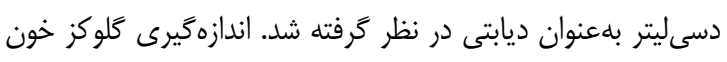

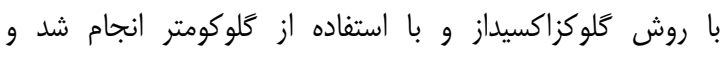

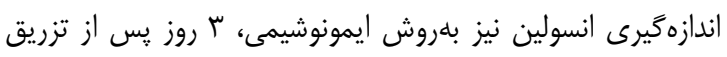

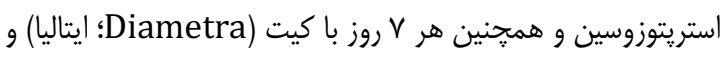

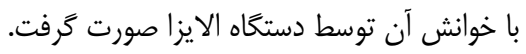

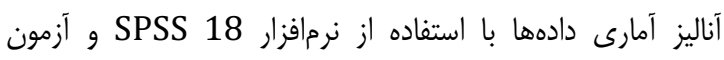

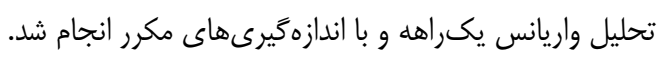

\section{يافتهها}

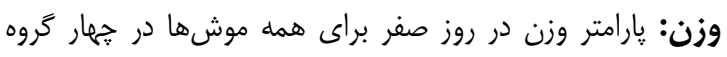

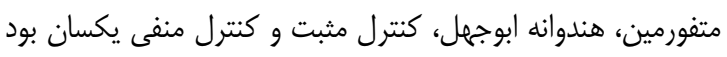

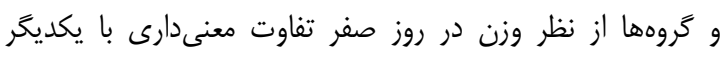

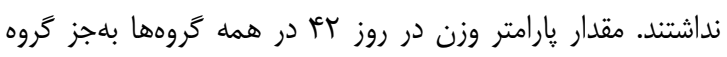

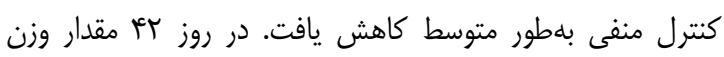

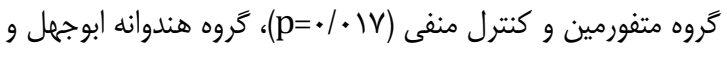

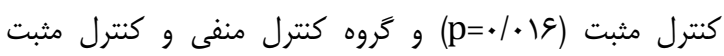

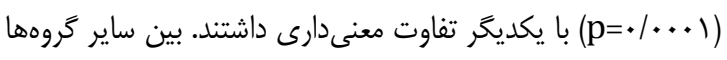

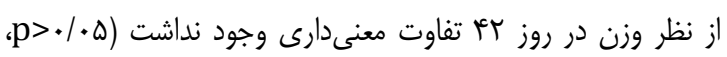

جدول ().

انسولين: در مورد مقدار انسولين در روز صفر و روز ّا، بين تمام

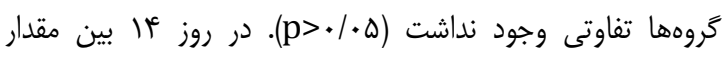

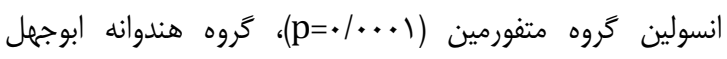
(p=|•l) )

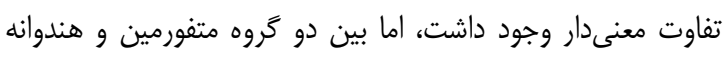

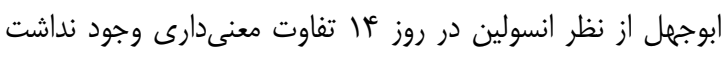

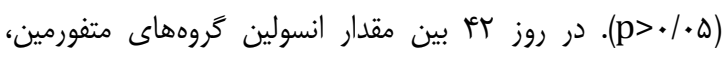

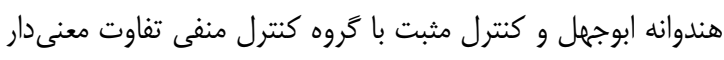

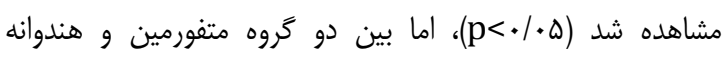

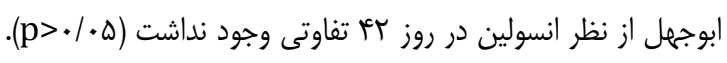

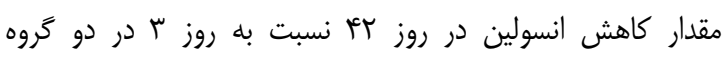

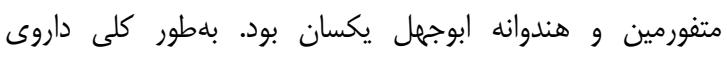

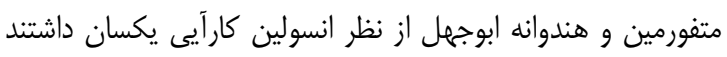

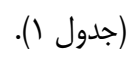

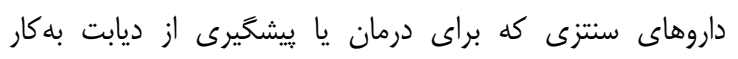

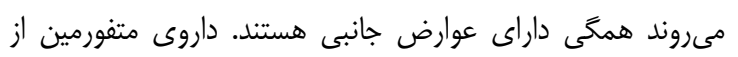

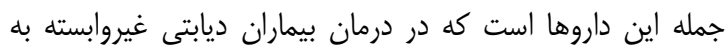

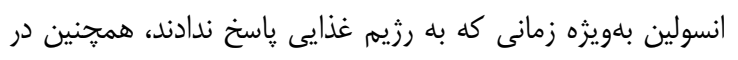

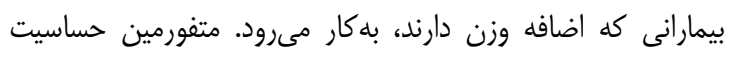

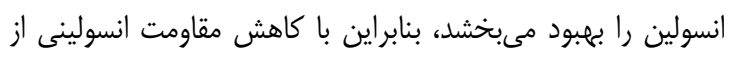

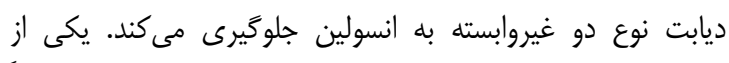

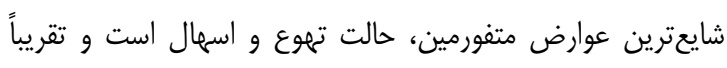

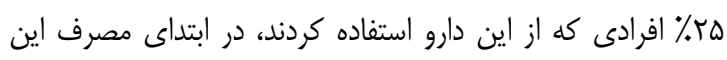

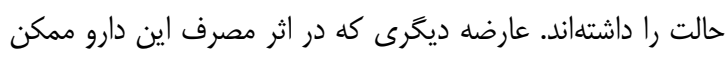

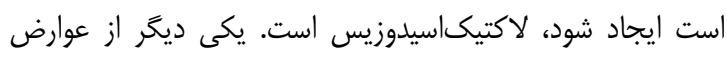

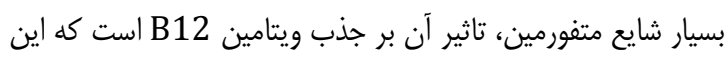

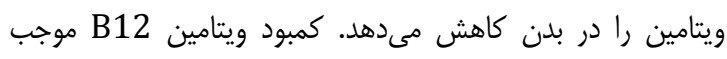

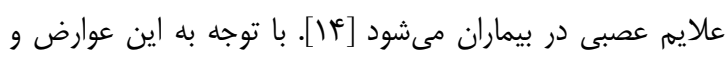

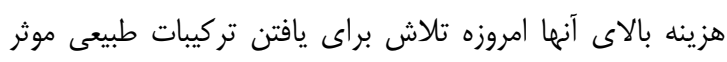
افزايش يافته است. اين مطالعه با هدف مقايسه اثرات ضدديابتى يودر ميوه هندوانه ابوجهل با داروى متفورمين بر ميزان كلوكز خون انجام شد.

\section{مواد و روشها}

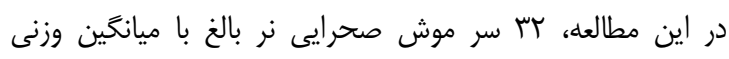

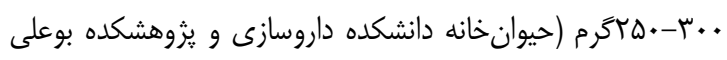

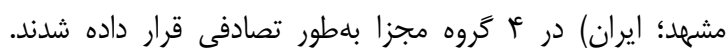

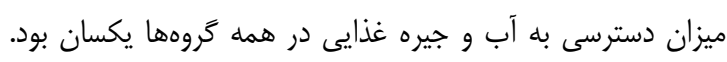

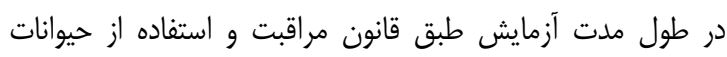

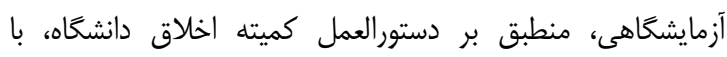

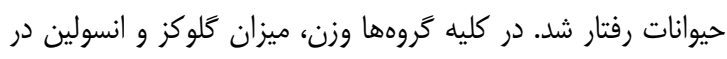

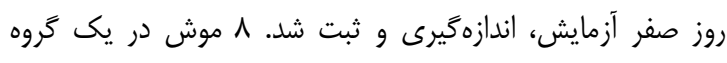

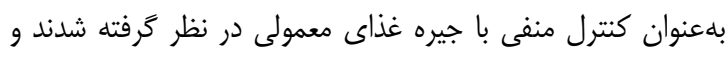

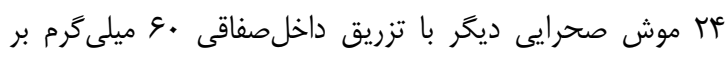

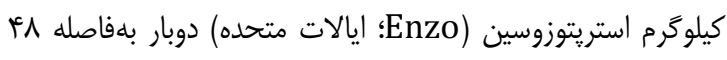

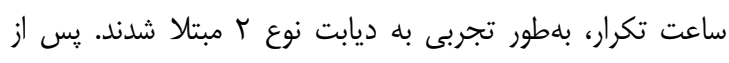

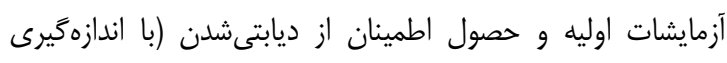

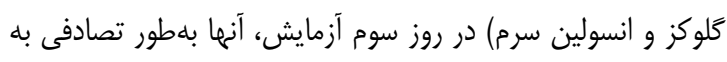

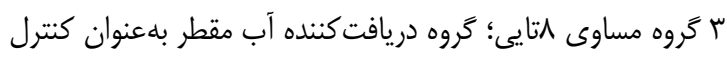

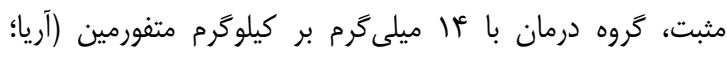

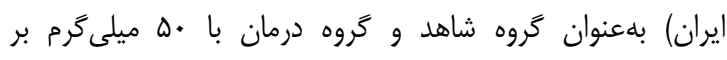

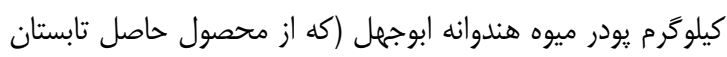

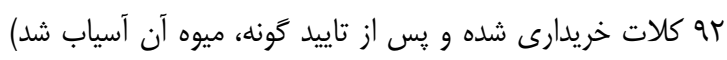

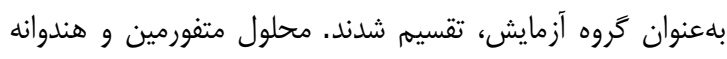

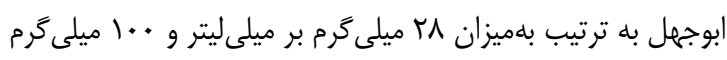

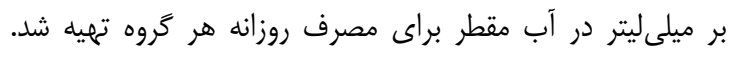


جدول () مقايسه متغيرهاى وزن، ميزان انسولين و كلوكز خون در روزهاى مختلف در كروههاى مورد مطالعه

\begin{tabular}{|c|c|c|c|c|}
\hline كنترل منفى & كنترل مثبت & هندوانه ابوجهل & متفورمين & شاخص \\
\hline & & & & وزن (كرم) \\
\hline$r F \cdot / l f \pm I I / \cdot r$ & $M F V / \Lambda \Delta \pm Q / \Delta 1$ & $r M T / F T \pm r \Delta / \& \Lambda$ & $r \Delta \cdot / V I \pm I Q / \varepsilon \varphi$ & روز صفر \\
\hline$T F \Delta / I F \pm I I / M$ & $|\wedge \varepsilon / \Delta V \pm 1| / 9 \Delta$ & $Y / N / \wedge \Delta \pm \mid N / I S$ & $\mid \Lambda V / T \Lambda \pm M T / r$. & روز \\
\hline \multirow[t]{2}{*}{$+Q / \cdot \cdot \pm r \cdot|r|$} & $-81 / r \Lambda \pm 19 / 19$ & $-I F / \Delta V \pm r V / a r$ & 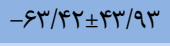 & اختلاف روز صفر و بr \\
\hline & & & & انسولين (ميكروواحد بر ميلىليتر) \\
\hline$\Delta / \wedge \Delta \pm \Gamma / ৭ \Delta$ & $\Delta / \Delta \cdot \pm \cdot / V)$ & $\Delta / r \Psi \pm r / I \varepsilon$ & $r / \Lambda) \pm r / \Delta Q$ & روز صفر \\
\hline $1 / \Delta T \pm \cdot / T V$ & $r / \wedge \pm 1 / / r$ & $r / T \cdot \pm \cdot / \Lambda t$ & $r / F T \pm r / l$. & (روز \\
\hline$|Q / \varepsilon| \pm Y / F Y$ & $1 / \Lambda \cdot \pm \cdot / \mu r$ & $\varphi / \cdots \pm \psi / r \Delta$ & $r / q \cdot \pm r / q q$ & روز \\
\hline \multirow[t]{2}{*}{$r N / T E \pm q / Y r$} & $r / \Lambda V \pm r / V \varepsilon$ & $0 / \cdot 1 \pm 1 / 4 \pi$ & $r / \Lambda \varepsilon \pm r / \Lambda r$ & ا روز Tr \\
\hline & & & & كَلوكز (ميلى كرم بر دسىليتر) \\
\hline$\|\varepsilon / V I \pm\| / \| V$ & $|r N / V| \pm T r / F V$ & $\|Q /\| F \pm \| r / I r$ & $\mid r q / \cdots \pm r \Delta / r$. & روز صفر \\
\hline$|r \pi / \cdot \cdot \pm \|| \mid / Q V$ & $T K Y / \Lambda \Delta \pm M F / \cdot 1$ & $M F N / \Delta V \pm M T / F \varepsilon$ & $r M N / \Delta V \pm T q / \cdot r$ & روز \\
\hline$|r V / \Lambda \Delta \pm| f / \cdot 1$ & KNI/ETEMT/rE & $\mathrm{TDT} / \Lambda \Delta \pm \mid \varepsilon / \cdot r$ & 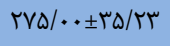 & | روز \\
\hline 9) $/ \Delta V \pm 1 / \mathrm{VI}$ & $r \wedge I / r \backslash \pm \Delta r / r r$ & $r \cdot 1 / 4 t \pm r \cdot / r t$ & $r F I / \& T \pm Q H / N E$ & إوز \\
\hline ११//I $\pm \Lambda / \cdot V$ & rงQ/VINET/\&\& & $109 / K^{2}+F \mid / 19$ & $\mid \Lambda Y / \Delta V \pm Y \cdot / r \varepsilon$ & روز Tr \\
\hline
\end{tabular}

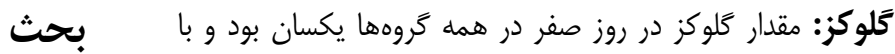

ديابت يك اختلال متابوليك ييجيده با علل كَوناكون است كه

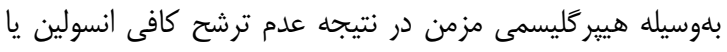

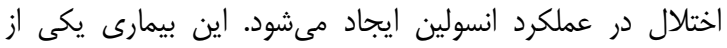

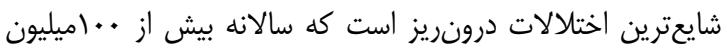

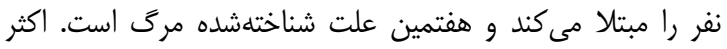

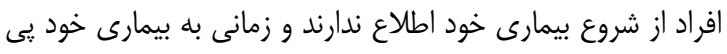

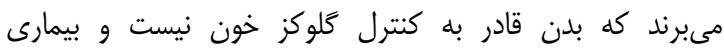

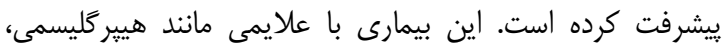

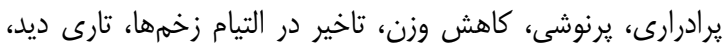

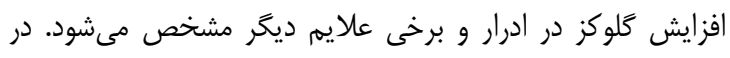

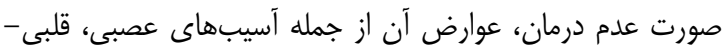

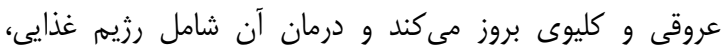

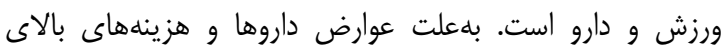

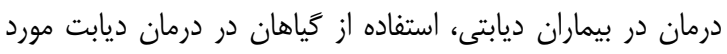

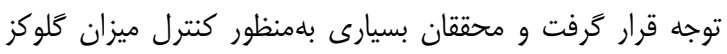

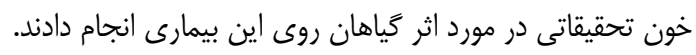

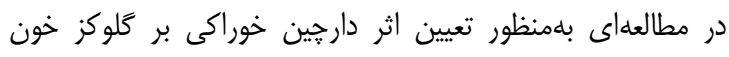

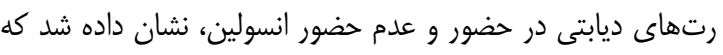

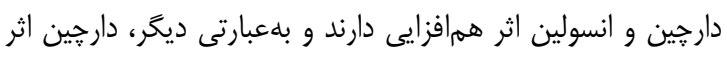

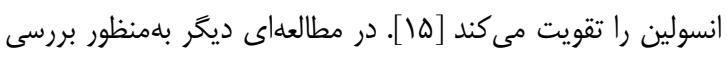

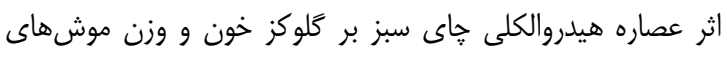

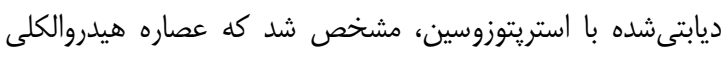

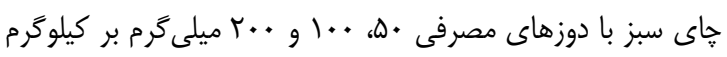

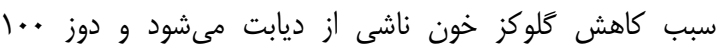

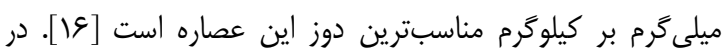

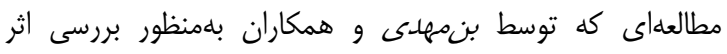

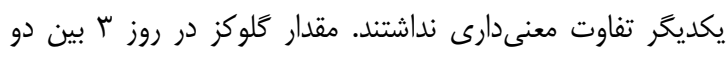

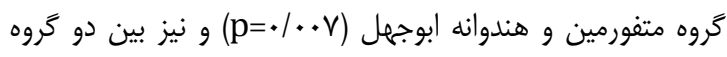

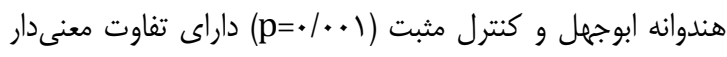

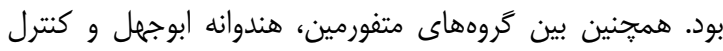

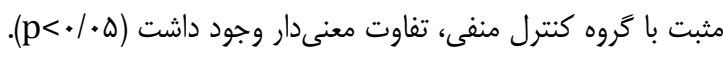

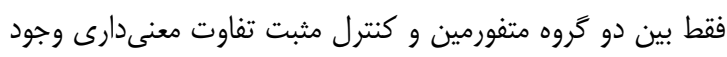

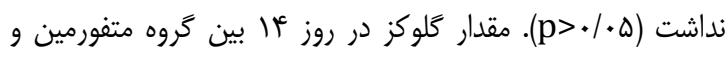

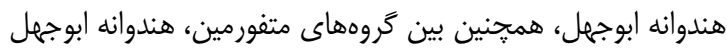

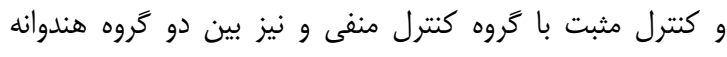

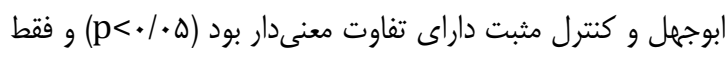

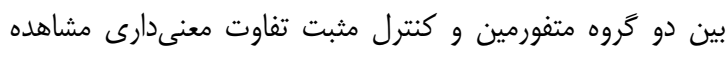

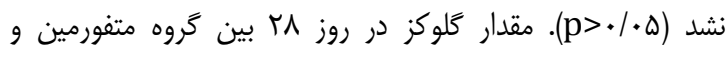

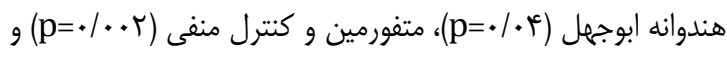

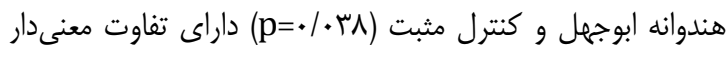

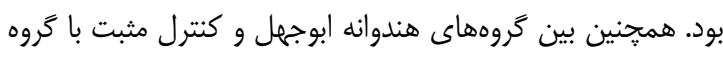

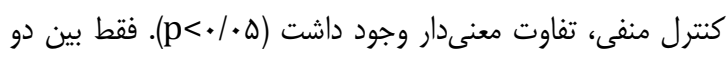

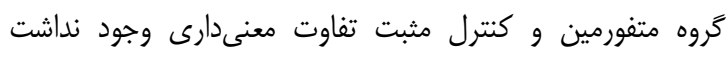

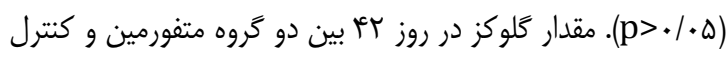

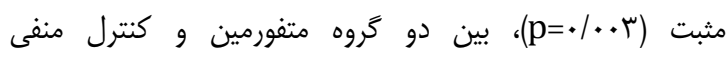
(p=/·^)

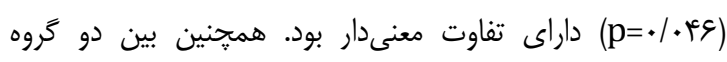

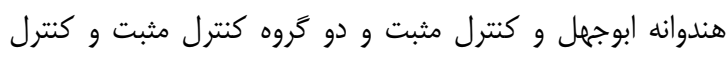

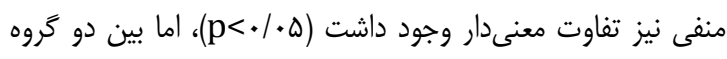

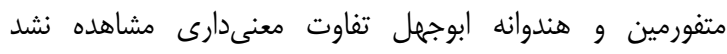

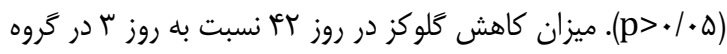

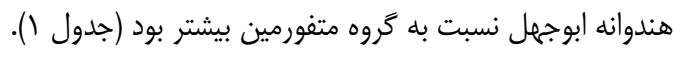

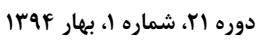
فصل نامه افق دانش 
مقايسه تاثير خوراكى يودر ميوه هندوانه ابوجهل با متفورمين بر ميزان كلوكز خون موشهاى صحرايى نر ديابتىشده با استريتوزوسين 11

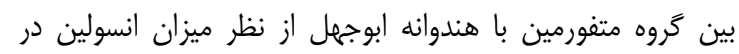

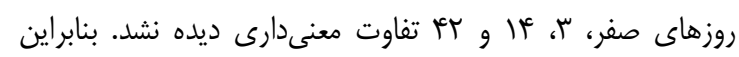

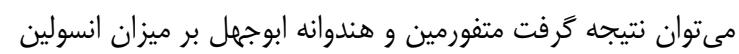
اثر يكسانى دارند.

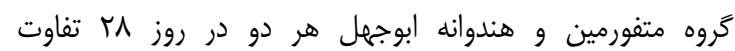

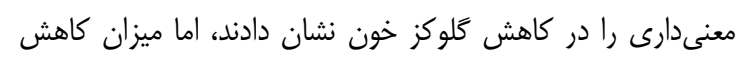

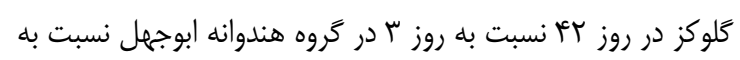

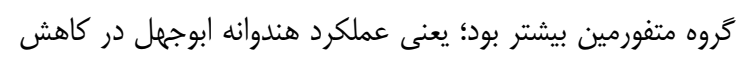

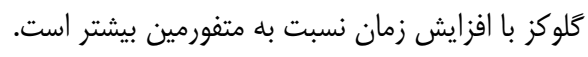

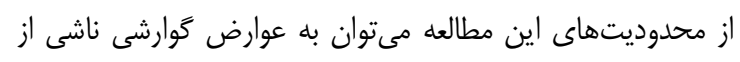

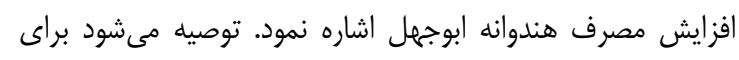

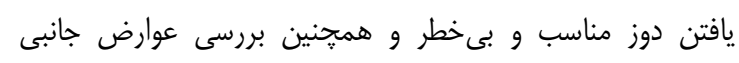

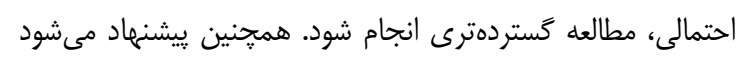

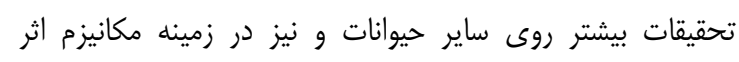

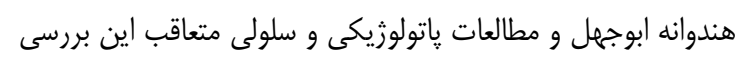

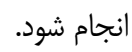

نتيجه كَيرى

يودر ميوه هندوانه ابوجهل در مقايسه با داروى متفورمين تا حدود

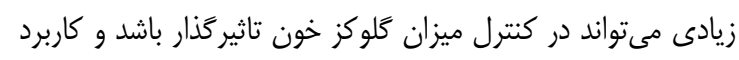

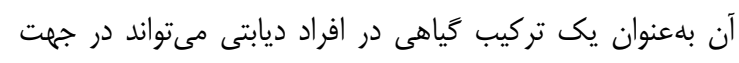

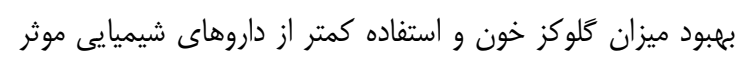

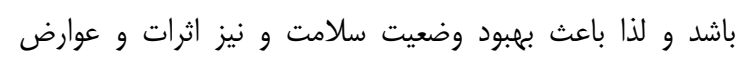
كمترى از داروها شود.

تشكر و قدردانى: دانشخاه فردوسى مشهر بهدليل فراهمسازى

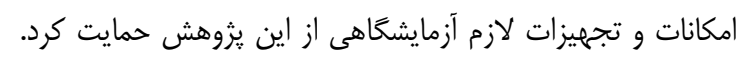

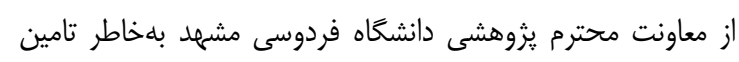

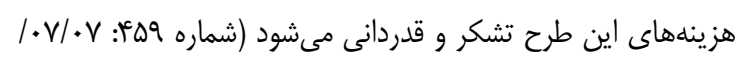
rوسا ) مشرهد، ايران.

تاييديه اخلاقى: اين مطالعه، طبق قانون مراقبت و استفاده از حيوانات آزمايشگاهى، منطبق بر دستورالعمل كميته اخلاق دانشگاه

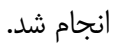

تعارض منافع: موردى توسط نويسندكان گزارش نشده است.

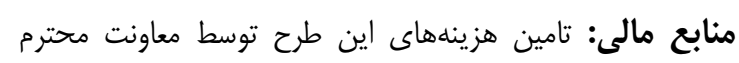
يثرهشى دانشگاه فردوسى مشهر انجام شده است.

منابع

1- Dallak M, Bin-Jaliah I. Antioxidant activity of Citrulus colocynthis pulp extract in the RBC's of alloxan- induced diabetic rats. Pak J Physiol. 2010;6(1):1-5.

2- Arise RO, Malomo SO, Adebayo JO, Igunnu A. Effects of aqueous extract of Eucalyptus globules on lipid peroxidation and selected enzymes of rat liver. J Med Plants Res. 2009;3(2):77-81.

Q Horizon Med Sci

Vol. 21, Iss. 1, Spr 2015

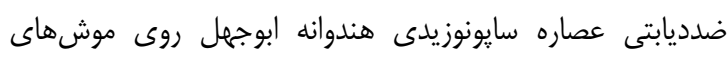

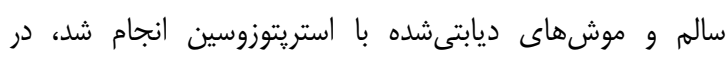

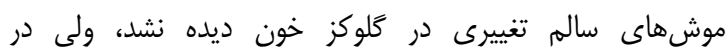

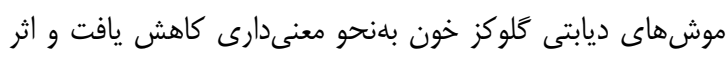

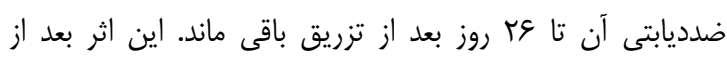

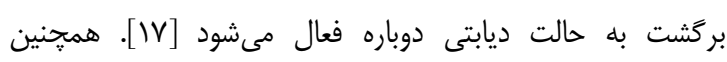
بلهنظور بررسى اثر آنتى ديابتيك عصاره آبى ميوه هندوانه ابوجهل

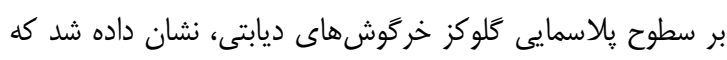

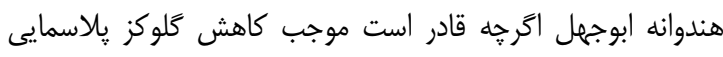

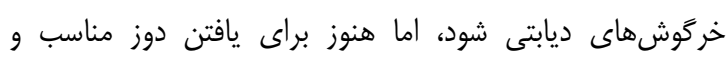

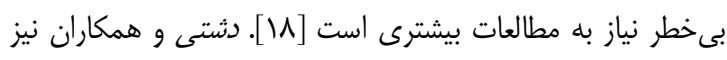

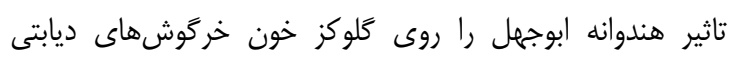

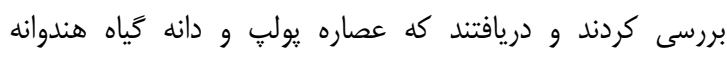

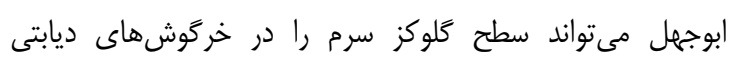
كاهش دهد [19]. مطالعات ديخر نشان داد كه مصرف خوراكى عصاره هندوانه ابوجهل روى كاهش استرس اكسيداتيو ناشى از

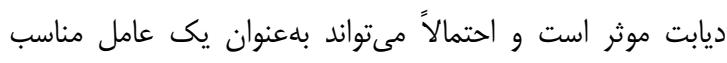

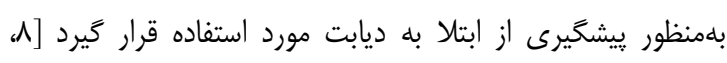

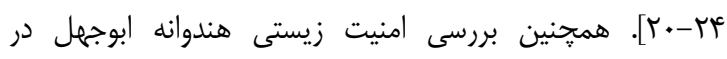

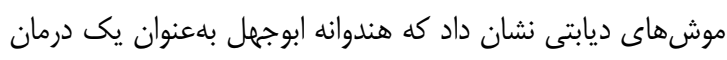

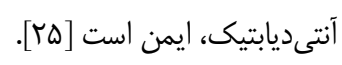
براساس مطالعه حاضر بلمنظور كنترل كلوكز خون در موشهاي استي

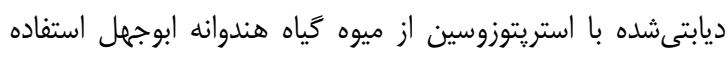

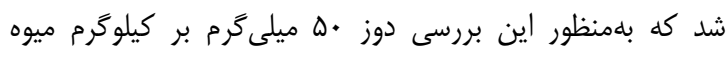

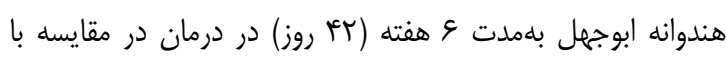

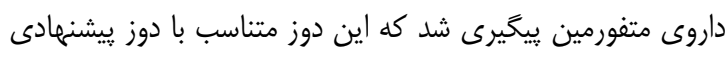
مطالعات قبلى است. با توجه به نتايج حاصله و در راستاى تحقيقات

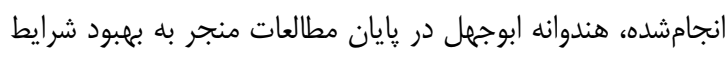

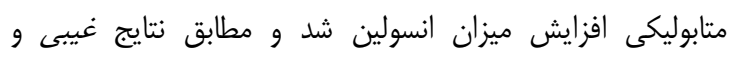

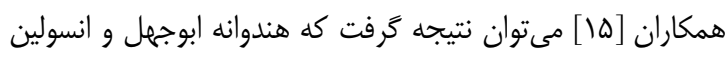

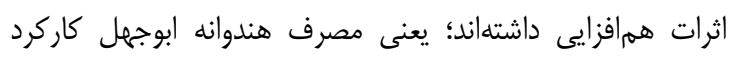

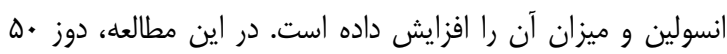
ميلى

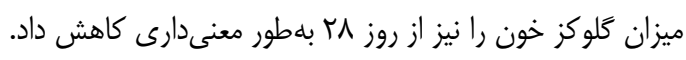

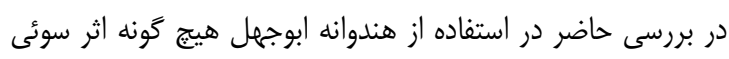

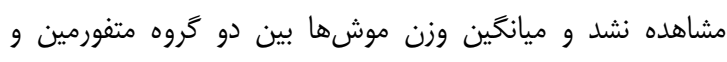

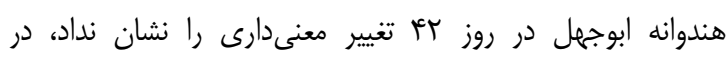

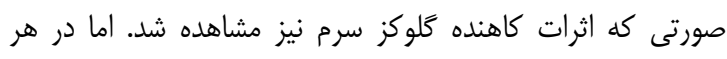

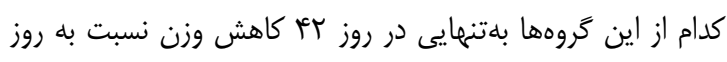

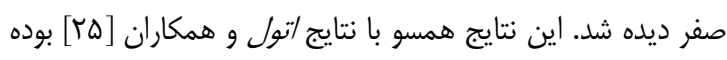

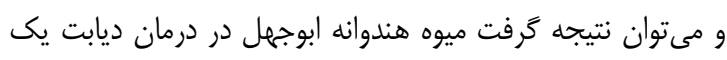
داروى ايمن است. 
15- Gheibi N, Parvizi MR, Jahani Hashemi H. The effect ofcinnamon on glucose concentration of diabetic rats in presence or absence of insulin. J Ghazvin Univ Med Sci. 2005;9(3):3-7. [Persian]

16- Mehdizade M, Hosseini SA, Ebrahiminia F, Elahi A, Fallah Hosseini $H$, Azizi $M$, et al. Effect of green tea (Camellia sineisis L.) extract on blood glucose and body weight in male induced diabetic rats. J Gorgan Univ Med Sci. 2009;11(1):8-12.

17- Benmehdi H, Azzi R, Djaziri R, Lahfa F, Benariba N, Tabti B. Effect of saponosides crude extract isolated from Citrullus colocynthis (L.) seeds on blood glucose level in normal and streptozotocin induced diabetic rats. J Med Pharm Sci. 2011;5(31):6864-8.

18- Zamani M, Rahimi AO, Mahdavi R, Nikbakhsh M, Jabbari MV, Rezazadeh $\mathrm{H}$, et al. Assessment of antihyperlipidemic effect of Citrullus colocynthis. Revista Brasileira de Farmacognosia. 2007;17(4):492-6.

19- Dashti N, Zamani M, Mahdavi R, Ostad Rahimi A. The effect of citrulluscolocynthis on blood glucose profile level in diabetic rabbits. J Jahrom Univ Med Sci. 2012;9(1):27-32.

20- Zareei Mahmoudabadi AB, Fallahhossini F, Sharifabady R, Nourouzzadeh A, Imani H, Ghoshouni H. The effect of Citrullus colocynthis Extract on the preventing/reducing streptozotocin-induced diabetes in rat. Kowsar Med J. 2007;12(1):13-20.

21- Goldfain D, Lavergne A, Galian A, Chauveinc L, Prudhomme F. Peculiar acute toxic colitis after ingestion of colocynth: A clinicopathological study of three cases. Gut. 1989;30(10):1412-8.

22- Fallah Huseini H, Heshmat R, Larijani B, Fakhrzadeh H, Jafariazar Z, Darvishzadeh F, et al. The clinical investigation of Citrullus colocynthis (L.) Schrad. fruit in treatment of type II diabetic patients; a randomized, double-blind, placebo-controlled study. J Med Plants. 2006;1(Suppl2):31-5. [Persian]

23- Larrey D. Hepatotoxicity of herbal remedies. J Hepatol. 1997;26(Suppl1):47-51.

24- Adam SE, Al-Yahya MA, Al-Farhan AH. Combined toxicity of Cassia senna and Citrullus colocynthis in rats. Vet Hum Toxicol. 2001;43(2):70-2.

25- Atole SK, Jangde CR, Philip P, Rekhe DV, Aghav DV, Waghode HJ, Chougule AM. Safety evaluation studies of Citrullus colocynthis for diabetes in rats. Vet World. 2009;2(11):423-5. r آ نسيم مجاز دلفاردى و همكاران

3- Zahrani HS, Amer KH. A comparative study on citrullus colocynthis plants grown in different altitudinal location in Saudi Arabia. American-Eurasian J Sci Res. 2006;1(1):1-7.

4- Ziyyat A, Legssyer A, Mekhfi H, Dassouli A, Serhrouchni M, Benjelloun W. Phytotherapy of hypertension and diabetes in oriental Morocco. J Ethnopharmacol. 1997;58(1):45-54.

5- Rahbar AR, Nabipour I. The hypolipidemic effect of Citrullus colocynthis on patients with hyperlipidemia. Pak J Biol Sci. 2010;13(24):1202-7.

6- Mardani M, Rezapour S, Rezapour P. The role of Citrullus colocynthis in the control and treatment of diabetes mellitus. J Nurs Midwifery, Lorestan Univ Med Sci. 2008;4(10-11):37-44. [Persian]

7- Shafaei H, Soleimani Rad J, Mahdavi R, Ostad Rahimi AR, Rezazadeh $\mathrm{H}$, Argani $\mathrm{H}$, et al. The potentiating effects of Citrullus colocynthis extract on immune system. Med J Tabriz Univ Med Sci. 2007;29(2):77-82. [Persian]

8- Khalil M, Mohamed G, Dallak M, Al-Hashem F, Sakr H, Refaat A, et al. The effect of Citrullus colocynthis pulp extract on the liver of diabetic rats a light and scanning electron microscopic study. American J Biochemistry Biotechnology. 2010;6(3):155-63.

9- Marzouk B, Marzouk Z, Décor R, Edziri H, Haloui E, Fenina N, et al. Antibacterial and anticandidal screening of Tunisian Citrullus colocynthis Schrad. from Medenine. J Ethnopharmacol. 2009;125(2):344-9.

10- Benalla W, Bellahcen S, Bnouham M. Antidiabetic medicinal plants as a source of alpha glucosidase inhibitors. Curr Diabetes Rev. 2010;6(4):247-54.

11- Barri ME, Onsa TO, Elawad AA, Elsayed NY, Wasfi IA, Abdul-Bari EM, et al. Toxicity of five Sudanese plants to young ruminants. J Comp Pathol. 1983;93(4):559-75.

12- Dehghani F, Panjehshahin MR. The toxic effect of alcoholic extract of Citrullus colocynthis on rat liver. Iranian J Pharmacol Ther. 2006;5(2):117-19.

13- Mahmoodi M, Sayyadi AR, HosseiniZijoud SM, Hajizadeh MR, Kazemi Arababadi M, Asadikaram GR, et al. Survey on the effects of different concentrations of Citrullus colocynthis fruit powder on some of the blood biochemical factors in normal and diabetic male rats. J Rafsanjan Univ Med Sci. 2012;11(1):11-20. [Persian] 14- Rendell M. Dietary treatment of diabetes mellitus. New England J Med. 2000;342(19):1440-1. 